\title{
Abundant $b$-Type Ions Produced in Electron Capture Dissociation of Peptides Without Basic Amino Acid Residues
}

\author{
Haichuan Liu* and Kristina Håkansson \\ Department of Chemistry, University of Michigan, Ann Arbor, Michigan, USA
}

\begin{abstract}
We have investigated electron capture dissociation (ECD) of doubly protonated peptides with few or no basic amino acid residues (BAARs). For peptides containing one His, abundant $b$-type ions were only found when His was located adjacent to the N-terminus. Interestingly, $b$-type ions, particularly $b_{5}{ }^{+}$, were found to be the dominant product ions in ECD of peptides without BAARs. Fragmentation patterns of luteinizing hormone releasing hormone (LHRH) and vasopressin (VP), containing one Arg and one His, respectively, were compared to those of $\mathrm{Q}^{8}$-LHRH and oxytocin (OT) in which the BAAR is replaced with a non-BAAR. More $b$-type ions were found for $\mathrm{Q}^{8}$-LHRH and OT than for LHRH and VP. We also performed ECD of melittin and found no $b$-type ions from ECD of the $4+$ charge state; however, many low abundance $b$-type ions were produced in ECD of the $5+$ charge state. Possible mechanisms for the formation of $b$-type ions are discussed and we propose that such ions are formed as a consequence of protons being located at backbone amide nitrogens. (J Am Soc Mass Spectrom 2007, 18, 2007-2013) @ 2007 American Society for Mass Spectrometry
\end{abstract}

$\mathrm{E}$ lectron capture dissociation (ECD), first introduced in 1998 [1], is a tandem mass spectrometry (MS/MS) technique that utilizes reactions between multiply charged analyte cations and low-energy $(<1 \mathrm{eV})$ electrons. ECD of multiply protonated peptides results in preferential cleavage of backbone $\mathrm{N}-\mathrm{C}_{\kappa}$ bonds, forming $c^{\prime}$ - and $z^{\circ}$-type product ions, which are complementary to the $b$ - and $y^{\prime}$-type ions observed in traditional MS/MS processes, such as collision-activated dissociation (CAD) [1-3]. ECD has been applied to a variety of biological molecules, including peptides [1-3], proteins [1-3], oligonucleotides [4], oligosaccharides [5-6], and a siderophore [7]. In particular, ECD has been found to be highly useful for analyzing peptides and proteins containing post-translational modifications (PTMs) $[2,3]$ because labile PTMs can be retained on ECD fragments, thereby allowing their facile localization. Despite the broad applicability of ECD for structurally characterizing biological molecules, its detailed mechanism is still an active controversy for both experimentalists and theorists [8-33]. For peptides, electron capture is mainly thought to occur either at a charge center $\left(\mathrm{H}^{+}\right.$or metal cations) followed by hydrogen transfer $[10,27,28]$, at a backbone amide group followed by proton transfer [19, 23, 24], or at a neutral hydrogen bond followed by hydrogen transfer

Address reprint requests to Dr. K. Håkansson, Department of Chemistry, University of Michigan, 930 North University Ave., Ann Arbor, MI 481091055, USA. E-mail: kicki@umich.edu

* Current address: Biomolecular Resource Center Mass Spectrometry Facility and Department of Cell and Tissue Biology, University of California, San Francisco, 513 Parnassus Ave., San Francisco, CA 94143-0512, USA.
[22]. Recent work by Turecek and coworkers suggests that both the two former mechanisms may be in operation depending on the nature of the charge carrier [24, 29]. The question whether the backbone $\mathrm{N}-\mathrm{C}_{\omega}$ bond cleavage observed in ECD of peptides is a result of a nonergodic or ergodic process is also debated. Computational and experimental support for both sides of the argument have been presented [1, 10, 26, 32, 34]. $\mathrm{O}^{\prime}$ Connor and coworkers proposed that the initial $\mathrm{N}-\mathrm{C}_{\kappa}$ bond cleavage is followed by a radical cascade [17]. The same group has shown evidence for long-lived radical intermediates comprising non-covalent complexes of $c^{\prime}$ and $z^{\bullet}$ ions in which multiple hydrogen migrations may occur $[9,18,21]$. Further support for such hydrogen migrations has been reported by Zubarev and coworkers [35].

Recently, attention has been directed to unconventional ECD fragments, mainly $b$-type ions $[8,11,14,16$, 25]. Cooper [11] was the first to investigate the presence of $b$-type ions in ECD. She found that their presence is dependent on the type of charge carrier (lysine \{Lys\} versus arginine $\{\mathrm{Arg}\})$ and the peptide structure. Specifically, Lys-containing peptides yielded more $b$-type ions than Arg-containing peptides in ECD. In addition, abundant $b$-type ions were found for a peptide $(\mathrm{H}-$ LLLLPLLLLK-OH) with Lys at the C-terminus, but such product ions were absent in ECD of the peptide H-KLLLLPLLLL-OH with Lys at the N-terminus. Two possible mechanisms were proposed for explaining the presence of $b$-type ions in ECD. First, Uggerud et al. [8] have performed ab initio direct dynamics calculations on several model systems and found that $b$-type ions 
can be directly formed upon electron capture if a backbone amide nitrogen is protonated. Second, $b$-type ions may be formed from vibrationally excited evenelectron charge-reduced precursor ions, i.e., $(\mathrm{M}+\mathrm{H})^{+}$, formed following hydrogen atom loss from $(\mathrm{M}+2 \mathrm{H})^{+}$ -. The latter mechanism was also evoked by Haselmann and Schmidt [14] to explain the presence of $b$-type ions in ECD of a doubly acetylated Lys-containing peptide. Recently, Marshall and coworkers [25] have investigated the effect of charge location in ECD. They found increased formation of $a$-type ions for the free-acid form of peptides containing basic residues near the N-terminus compared with their C-terminally amidated forms. These authors suggested that amide nitrogen protonation accounts for the presence of such ECD fragments.

All peptides studied previously contain at least one basic amino acid residue $(\mathrm{BAAR}=\mathrm{Arg}$, Lys, and histidine $\{$ His\}). To our knowledge, ECD of peptides without BAARs has not been explored in detail. Here, we present ECD of peptides with one His or without BAARs. ECD of peptides with one BAAR is also compared with ECD of the same peptide but with the BAAR replaced by a non-BAAR. Moreover, comparisons are made between ECD of the $4+$ and $5+$ charge states of melittin. Previously proposed mechanisms for the formation of $b$-type ions are also discussed in the context of these data.

\section{Experimental}

\section{Reagents and Sample Preparation}

The peptides used in this work can be divided into four groups. The first group consists of peptides containing His as the only BAAR, including $\alpha_{1}$-mating factor fragment 1-6 (MF = H-WHWLQL-OH), neuromedin B $\left(\mathrm{NMB}=\mathrm{H}-\mathrm{GNLWATGHFM- \textrm {NH } _ { 2 }}\right)$, and neuromedin $\mathrm{K}$ $\left(\mathrm{NMK}=\mathrm{H}-\mathrm{DMHDFFVGLM}-\mathrm{NH}_{2}\right)$. The second group includes three peptides without any BAAR, i.e., cholecystokinin fragment 26-33 (CCK = H-DYMGWMDF$\left.\mathrm{NH}_{2}\right)$, pEVNFSPGWGT-NH ${ }_{2}$ (= Pep1), and (pGlu ${ }^{5}$, D-Trp $\left.{ }^{7,9,10}\right)$-substance $P$ fragment 5-11 (TrpSP $=$ pEQWFWWM-NH $\mathrm{N}_{2}$ ). The third group contains two pairs of peptides, each having a peptide with one BAAR and its corresponding mutant without BAAR; luteinizing hormone releasing hormone $(\mathrm{LHRH}=\mathrm{pEHWSYGLRPG-}$ $\left.\mathrm{NH}_{2}\right)$ and its variant avian LHRH $\left(\mathrm{Q}^{8}-\mathrm{LHRH}=\mathrm{pEH}-\right.$ WSYGLQPG-NH $\left.{ }_{2}\right)$, and $\left(\mathrm{Arg}^{8}\right)$-vasopressin (VP = $\mathrm{H}-\mathrm{CYFQNCPRG- \textrm {NH } _ { 2 }}$, 1-5 disulfide bond) and oxytocin (OT $=\mathrm{H}-\mathrm{CYFQNCPLG-NH}, 1-5$ disulfide bond). Melittin is the only peptide in the fourth group. All peptides, except CCK, were purchased from Aldrich (St. Louis, MO). CCK was from Advanced ChemTech (Louisville, KY). These peptides were used without further purification. Multiply protonated peptide ions were generated by external electrospray ionization at 70 $\mu \mathrm{L} / \mathrm{h}$ (Apollo Ion Source; Bruker Daltonics, Billerica, MA) of solutions containing 2 to $10 \mu \mathrm{M}$ peptides in 49:49:2 methanol/water/acetic acid (all solvents except acetic acid were purchased from Fisher Scientific, Fair Lawn, NJ, while acetic acid was from Fisher Scientific, Pittsburgh, PA).

\section{Mass Spectrometry and Data Analysis}

All mass spectra were collected with an actively shielded 7 tesla Fourier transform ion cyclotron resonance (FT-ICR) mass spectrometer with a quadrupole front-end (APEX-Q, Bruker Daltonics) as previously described [19, 36]. Briefly, ions produced by electrospray ionization were mass-selectively externally accumulated (10-40 Th isolation window) in a hexapole for 1 or $2 \mathrm{~s}$, transferred via high voltage ion optics, and captured in the ICR cell by gated trapping. This accumulation sequence was looped six times to optimize precursor ion signal-to-noise $(\mathrm{S} / \mathrm{N})$ ratio before ECD. Further precursor ion isolation (required due to the presence of interfering ions with similar $\mathrm{m} / \mathrm{z}$ values as precursor ions of interest) was accomplished by correlated harmonic excitation fields (CHEF) [37] inside the infinity [38] ICR cell. As a result, mainly monoisotopic precursor ions were subject to MS/MS experiments. An indirectly heated hollow dispenser cathode [39] with inner and outer diameters of 3.5 and $7.6 \mathrm{~mm}$, respectively, provided the electrons. The cathode is mounted $88 \mathrm{~mm}$ behind the ICR cell. ECD was performed at a -1 $\mathrm{V}$ cathode bias voltage and an extraction lens voltage located in front of the cathode was kept at $1 \mathrm{~V}$. The electron irradiation time was 100 to $300 \mathrm{~ms}$. Collision activated dissociation (CAD) of mass-selected ions was performed in the external hexapole. The ion kinetic energy was increased by lowering the DC offset of the second accumulation hexapole from 0 to $\sim-6 \mathrm{~V}$ for $\mathrm{MF}$ and Pep1. The collision gas was argon at a pressure reading of 6 to $7 \times 10^{-6}$ torr (gauge calibrated for $\mathrm{N}_{2}$ ). All data were acquired with XMASS (version 6.1, Bruker Daltonics) in broadband mode from $\mathrm{m} / \mathrm{z}=200$ to 1600 with 256 to $512 \mathrm{k}$ data points and summed over 16 to 50 scans. Mass spectra were analyzed with the MIDAS analysis software [40]. A Hanning window function was applied and the data were zero-filled once before magnitude calculation. Internal frequency-tomass calibration was performed by Microsoft Excel with a two-term calibration equation [41]. The calculated masses of the precursor ion and the chargereduced species were used for calibration. For the ECD spectrum where no charge-reduced species was observed, external calibration was performed based on parameters from an internally calibrated ECD spectrum acquired under identical experimental conditions.

\section{Results and Discussion}

\section{ECD of Doubly Protonated Peptides Containing His as the Only BAAR}

Figure 1 shows product ion spectra resulting from ECD of three doubly protonated peptides (MF, NMB, and 
(a)
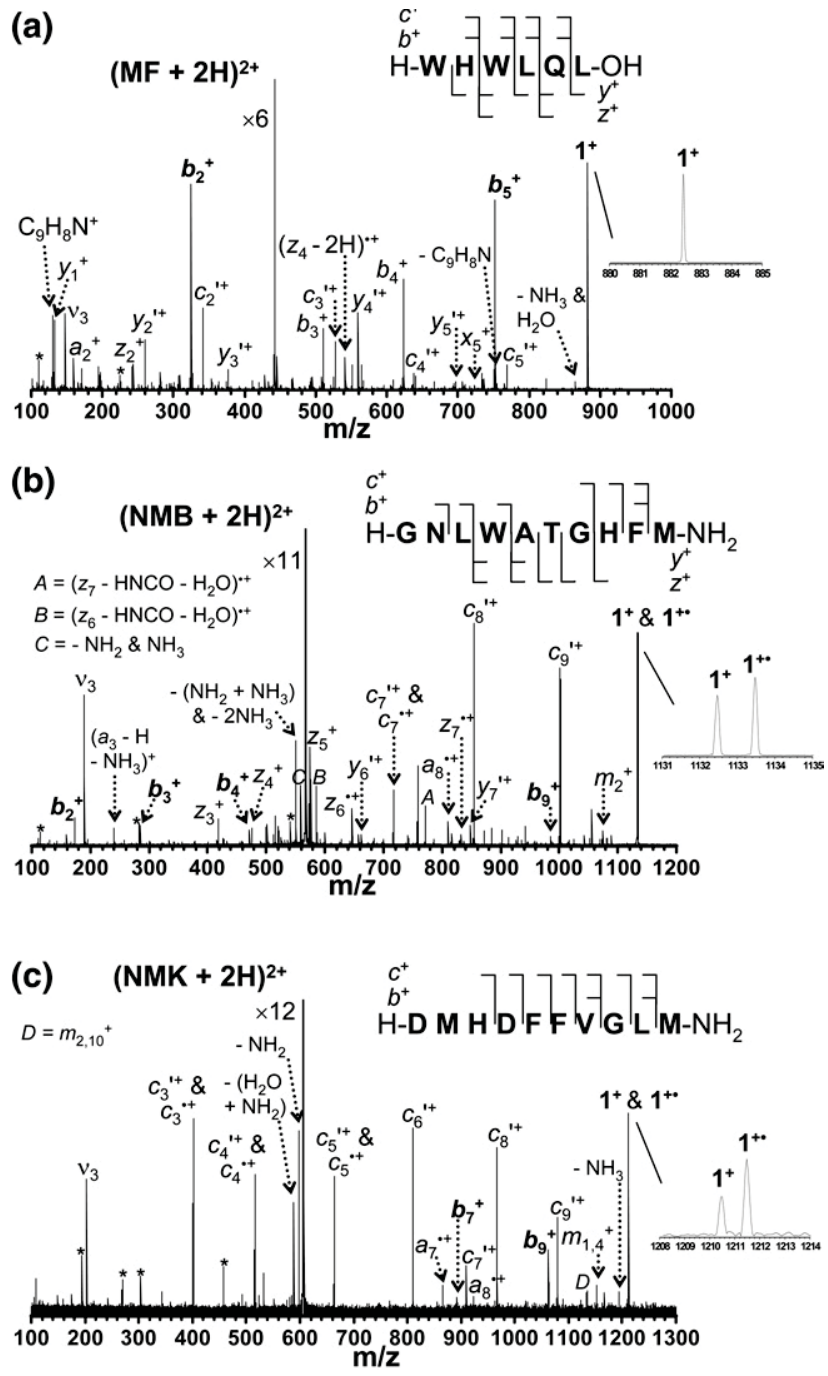

Figure 1. ECD spectra from $[\mathrm{MF}+2 \mathrm{H}]^{2+}(\mathbf{a}),[\mathrm{NMB}+2 \mathrm{H}]^{2+}(\mathbf{b})$, and $[\mathrm{NMK}+2 \mathrm{H}]^{2+}$ (c) $(300 \mathrm{~ms},-1 \mathrm{~V}$ cathode bias voltage, 16 to 50 scans). Electronic noise and product ions present before electron irradiation (as a result of peptide vibrational excitation) are labeled with asterisks, $\nu_{3}=$ third harmonic. Insets show expanded views of charge reduced species; " $\mathrm{m}_{n}$ " ions correspond to loss of the entire amino acid side chain at the $n$th residue from charge reduced precursor ions.

NMK) containing one His as the only BAAR. In these three peptides, the position of His is close to either the $\mathrm{N}$ - (for MF and NMK) or C-terminus (for NMB). ECD of $\mathrm{MF}, \mathrm{NMB}$, and NMK carrying two protons produced a mixture of different types of sequence ions, including $c$-, $z-, b-, y-$, and $a$-type ions for all three peptides, and one additional $x$-type ion $\left(x_{5}{ }^{+}\right)$for MF (Figure 1a). Although unconventional $b$-type ions are seen in all three cases, the relative abundance of $b$-type ions is dependent on the peptide sequence. As can be seen in Figure $1, b_{2}{ }^{+}$, and $b_{5}{ }^{+}$are the most abundant sequence ions in ECD of $(\mathrm{MF}+2 \mathrm{H})^{2+}$ (Figure 1a), whereas $b$-type ions are much less abundant than $c$-type ions in ECD of (NMB/NMK $+2 \mathrm{H})^{2+}$ (Figure $1 \mathrm{~b}$ and $\mathrm{c}$ ). The presence of abundant $b$-type ions for MF but not for NMB/NMK is somewhat unexpected because the sequence of MF is not significantly different than those of NMB and NMK. The only obvious difference between MF and the other two His-containing peptides is the position of His. In MF, His is adjacent to the N-terminal amino acid, while it is separated from the N-terminus by at least one amino acid residue in NMB and NMK. The peptide N-terminus and the side-chain of His are the two most basic sites in these peptides. For NMB and NMK, these two basic sites can be protonated simultaneously although charge repulsion could exist in the case of NMK. By contrast, simultaneous protonation of the N-terminus and the His side-chain in MF is less likely because it may induce significant Coulomb repulsion. Thus, only one of these two basic sites may be protonated in (MF + $2 \mathrm{H})^{2+}$. The other proton may be located at a backbone amide nitrogen or oxygen. As mentioned above, Uggerud and coworkers [8] have performed ab initio direct dynamics calculations on the fragmentation induced by electron attachment to model peptides. They concluded that ECD of backbone nitrogen-protonated peptides will produce $b$ - and $y$-type ions, whereas protonation involving solvation of a carbonyl oxygen is associated with the formation of $c$ - and z-type ions. Based on this conclusion, we believe that, in (MF + $2 \mathrm{H})^{2+}$, amide nitrogens constitute the second protonation site, thereby explaining the abundant $b$-type ions. To further investigate the formation of $b$-type ions in ECD, peptides without highly BAARs were characterized. The corresponding results are discussed in the following sections.

\section{ECD of Doubly Protonated Peptides Without BAARs}

Figure 2 presents ECD product ion spectra of three doubly charged peptides (CCK, Pep1, and TrpSP) without BAARs (Arg, Lys, and His). A variety of $b$-type ions are the major product ions in each case. For CCK and TrpSP (Figure 2a and c), all possible $b$-type ions $\left(b_{1}{ }^{+}\right.$ ions are typically not observed [42]) were detected in ECD and for Pep1 (Figure 2b), six out of nine possible $b$-type ions were produced in ECD. In comparison, only a few low abundance $c$ - and $z$-type ions were found in ECD of all three peptides. As discussed above, the presence of $b$-type ions in ECD has been associated with peptide protonation patterns $[8,11,14,16]$. For doubly protonated CCK, Pep1, and TrpSP without BAARs, at least one of the protons should be located at the peptide backbone, similar to the case of $(\mathrm{MF}+2 \mathrm{H})^{2+}$ (Figure 1a). Neutralization of this proton may result in formation of $b$-type ions, as discussed below. Interestingly, $b_{5}{ }^{+}$was found to be significantly more abundant than other $b$-type ions in all three cases, indicating a favored gas-phase conformation for doubly protonated CCK, Pep1, and TrpSP in which one proton is solvated by the five $\mathrm{N}$-terminal amino acid residues (the presence of abundant $b_{5}{ }^{+}$may also result from a compromise 
(a)
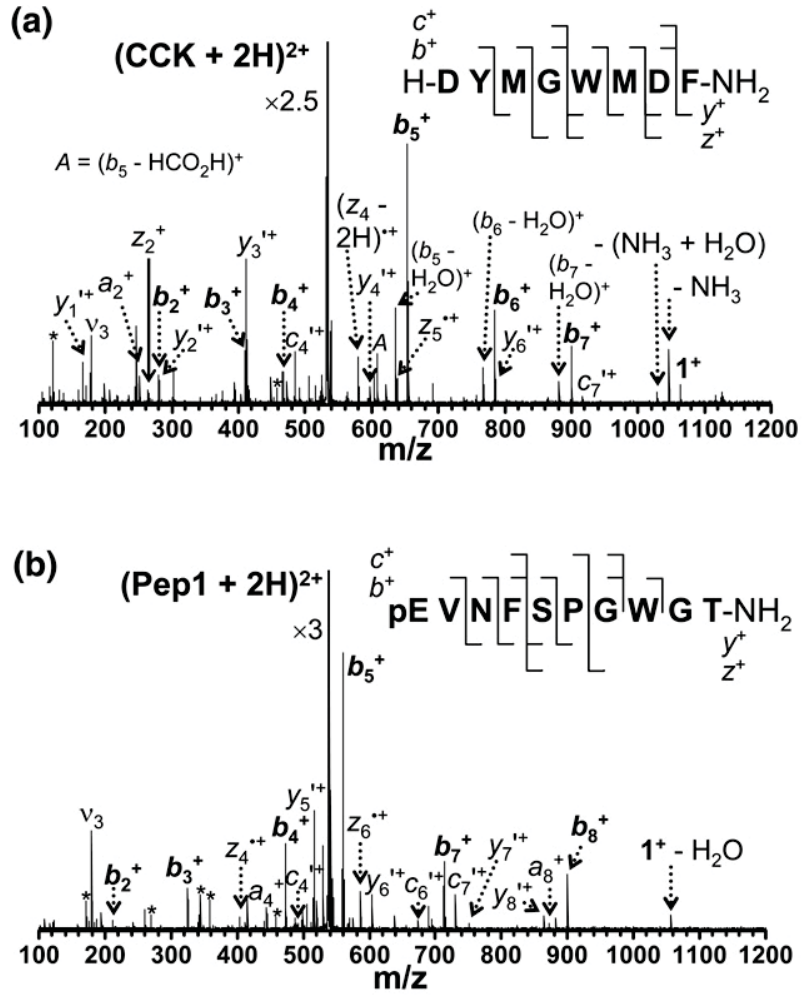

(c)

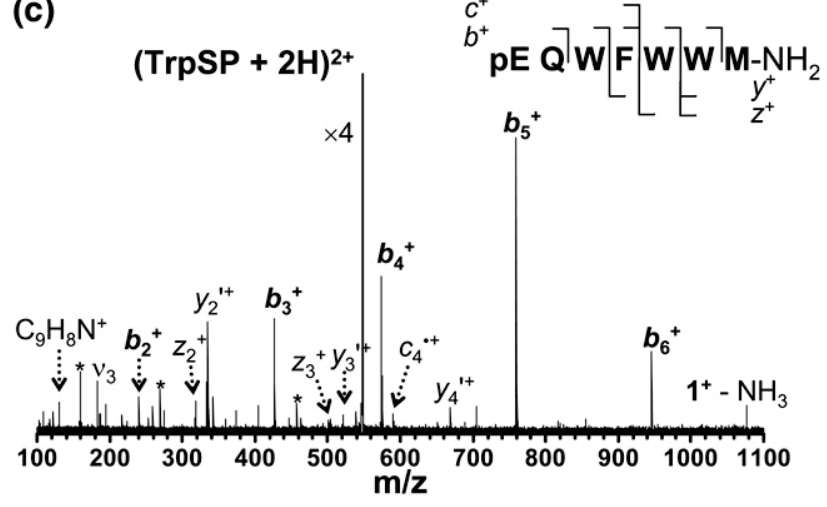

Figure 2. ECD spectra from $[\mathrm{CCK}+2 \mathrm{H}]^{2+}(\mathrm{a}),[\mathrm{Pep} 1+2 \mathrm{H}]^{2+}$ (b), and $[\operatorname{TrpSP}+2 \mathrm{H}]^{2+}$ (c) $(300 \mathrm{~ms},-1 \mathrm{~V}$ cathode bias voltage, 16 to 50 scans). Electronic noise and product ions present before electron irradiation (as a result of peptide vibrational excitation) are labeled with asterisks, $\nu_{3}=$ third harmonic.

between minimization of Coulomb repulsion and maximization of proton charge solvation by side chains and/or carbonyls). As found for MF (Figure 1a, inset), the odd-electron charge-reduced precursor ion was not detected for CCK, Pep1, and TrpSP. In fact, no chargereduced precursor ions were present in the ECD spectra of $(\mathrm{Pep} 1 / \operatorname{TrpSP}+2 \mathrm{H})^{2+}$, while only a minor evenelectron charge-reduced precursor ion $\left([\mathrm{M}+\mathrm{H}]^{+}\right)$was seen in ECD of $(C C K+2 \mathrm{H})^{2+}$. These results suggest that loss of a hydrogen radical from charge-reduced precursor ions following neutralization of the mobile proton at the peptide backbone could play a critical role in the formation of $b$-type ions, as proposed by Cooper [11] and by Haselmann and Schmidt [14].
Because $b$-type ions are commonly observed in vibrational excitation MS/MS, CAD of selected peptides (MF and Pep1) from group 1 and 2 was performed for the purpose of comparison with the ECD results. Figure 3 shows the resulting spectra. ECD and CAD of (MF + $2 \mathrm{H})^{2+}$ provided the same number of $b$-type ions $\left(b_{2-5}{ }^{+}\right)$. However, the relative abundance of $b_{2}{ }^{+}$to that of $b_{5}{ }^{+}$is significantly different in the two cases. In ECD of (MF + $2 \mathrm{H})^{2+}$ (Figure 1a), $b_{2}{ }^{+}$and $b_{5}{ }^{+}$are close to equally abundant, whereas the latter product ion is much more abundant than the former in CAD of $(\mathrm{MF}+2 \mathrm{H})^{2+}$ (Figure 3a). Doubly charged $b_{5}{ }^{2+}$ was also found to be a major product ion in CAD of $(\mathrm{MF}+2 \mathrm{H})^{2+}$. For Pep1, CAD (Figure $3 b$ ) resulted in fewer $b$-type ions than ECD (Figure 2b). In CAD, $b_{4}{ }^{+}$is the most abundant product ion, whereas $b_{5}{ }^{+}$is the dominant fragment in ECD (Figure 2b). These differences confirm that the formation of $b$ ions in ECD is not simply due to vibrational excitation but associated with the protonation pattern of the peptide, as mentioned above.

\section{ECD of Peptides Containing BAARs and Their Analogues with Fewer or No BAARs}

We also performed ECD of peptides containing BAARs and their analogues with fewer or no BAARs. Figure 4 shows product ion spectra resulting from ECD of VP and OT. The only difference between the sequences of $\mathrm{VP}$ and OT is that the second amino acid residue from the C-terminus is Arg in VP but Leu in OT, which thus
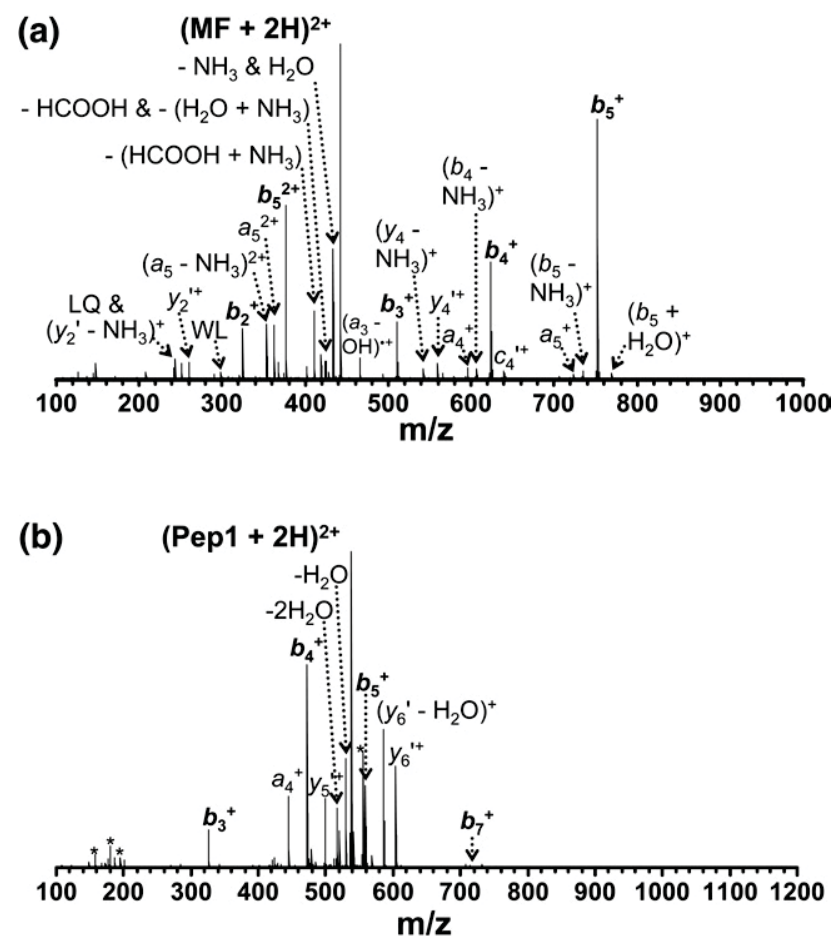

Figure 3. CAD spectra from $[\mathrm{MF}+2 \mathrm{H}]^{2+}$ (a) and $[\mathrm{Pep} 1+2 \mathrm{H}]^{2+}$ (b). Electronic noise and product ions present before electron irradiation (as a result of peptide vibrational excitation) are labeled with asterisks. 
(a)
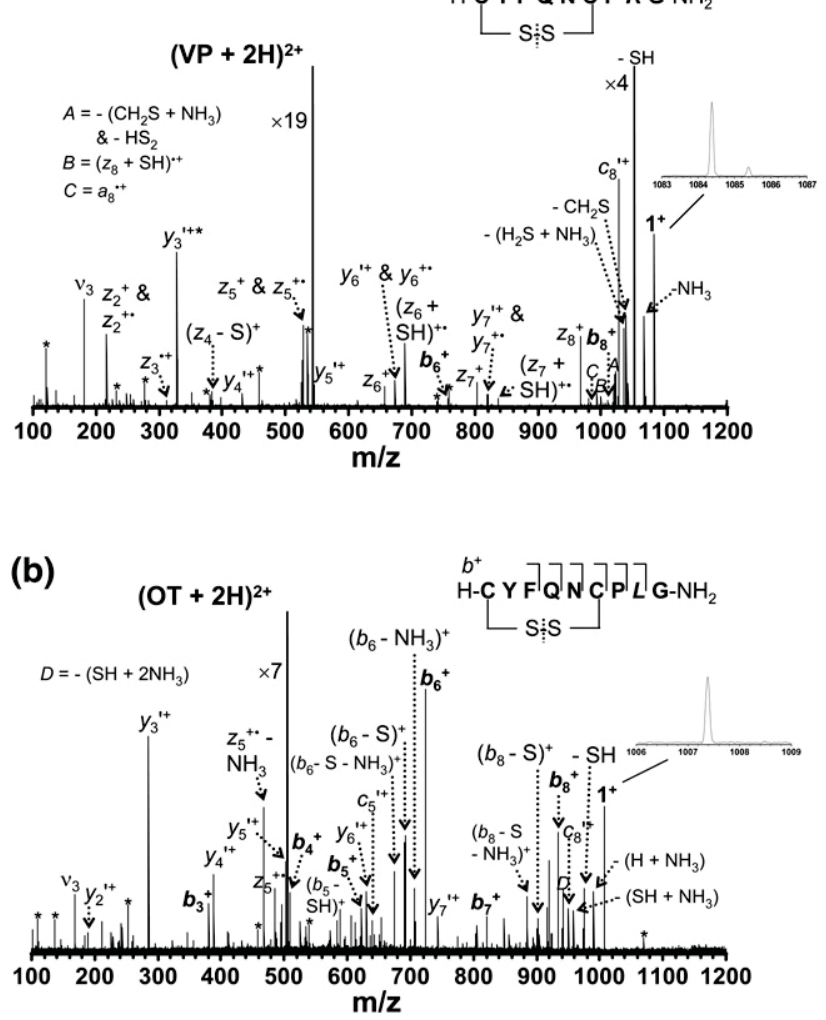

Figure 4. ECD spectra from $[\mathrm{VP}+2 \mathrm{H}]^{2+}$ (a) and $[\mathrm{OT}+2 \mathrm{H}]^{2+}$ (b) (300 ms, $-1 \mathrm{~V}$ bias voltage, 30 and 50 scans for VP and OT, respectively). Electronic noise peaks are labeled with asterisks, $\nu_{3}$ $=$ third harmonic. Insets show expanded views of charge reduced species.

contains no BAARs. As can be seen in Figure 4a, ECD of $(\mathrm{VP}+2 \mathrm{H})^{2+}$ produced $c_{8}{ }^{+}$as the dominant product ion along with some $z$-type ions $\left(z_{2}{ }^{\bullet+}-z_{8}{ }^{\bullet+}\right.$, except $\left.z_{4}{ }^{++}\right)$of moderate abundance whereas only two $b$-type ions $\left(b_{6}{ }^{+}\right.$ and $b_{8}{ }^{+}$) of very low abundance were observed. By comparison, all but two possible $b$-type ions were formed in ECD of $(\mathrm{OT}+2 \mathrm{H})^{2+}$ (Figure $\left.4 \mathrm{~b}\right)$, for which $b_{6}{ }^{+}$and $b_{8}{ }^{+}$are particularly abundant. Two $c$-type $\left(c_{8}{ }^{+}\right.$ and $\left.c_{5}{ }^{+}\right)$and one $z$-type ion $\left(z_{4}{ }^{+}\right)$were still seen for OT, but with much lower abundance than $b_{6}{ }^{+}$and $b_{8}{ }^{+}$. $y$-type ions were also found for both VP and OT, with $y_{3}{ }^{\prime+}$ being the most abundant one. A possible explanation for the presence of $y$ ions in ECD of $(\mathrm{OT}+2 \mathrm{H})^{2+}$ is that the C-terminal amide nitrogen is protonated while the other proton is located at one of the backbone amide nitrogens. Neutralization of the latter proton may result in fragmentation of backbone amide bonds, forming fragments carrying a proton at their C-termini, that is, $y$-type ions. Similar results as described here for VP/OT were observed in ECD of doubly protonated LHRH (pEHWSYGLRPG-NH ${ }_{2}$ ) and its variant $\mathrm{Q}^{8}$-LHRH (pEHWSYGLQPG-NH ${ }_{2}$ ) with fewer BAARs (see Supplementary Figure, which can be found in the electronic version of this article), further demonstrating the influence of BAARs in ECD.

\section{ECD of the $4+$ and $5+$ Charge States of Melittin}

The results discussed above show that abundant $b$-type ions are present in ECD of doubly protonated peptides if there is only one basic site. We propose that protonation of backbone amide nitrogens (due to the lack of basic sites) is responsible for the formation of $b$-type ions. From this reasoning, $b$-type ions would also be expected for higher charge states when protons outnumber the basic sites. To investigate this hypothesis, melittin carrying four and five protons, respectively, was fragmented with ECD. ECD of quadruply protonated melittin has been reported previously [43, 44]. Those data as well as our own results (data not shown) demonstrate that ECD spectra of quadruply protonated melittin are ripe with $c$ - and $z$-type ions and several $a$ and $y$-type ions are also observed. However, $b$-type ions are not produced in ECD of quadruply protonated melittin. Figure 5 shows an ECD spectrum of quintuply protonated melittin. Here, a plentitude of $b$-type ions are seen although their abundances are lower than those of $c$ - and $z$-type ions. This result indicates that the fifth proton for quintuply protonated melittin may be located either at a basic site, or at a backbone amide nitrogen. Zubarev and coworkers have investigated the charge state distributions of ECD product ions from triply, quadruply, and quintuply protonated melittin [43] and predicted the locations of 2,3, and 4 protons in those charge states, respectively. Three protonation sites in quadruply charged melittin were assigned to the $\mathrm{AV}^{4-5}$, IK $\mathrm{IK}^{20-21}$, and $\mathrm{RQ}^{24-25}$ residues, while four protons in quintuply-charged melittin were assigned to the $\mathrm{IG}^{2-3}, \mathrm{~T}(\mathrm{GLPALI})^{11-(17)}, \mathrm{R}^{22}$, and $\mathrm{Q}^{26}$ residues. We argue that the proton assigned to $\mathrm{AV}^{4-5}$ [43] could also

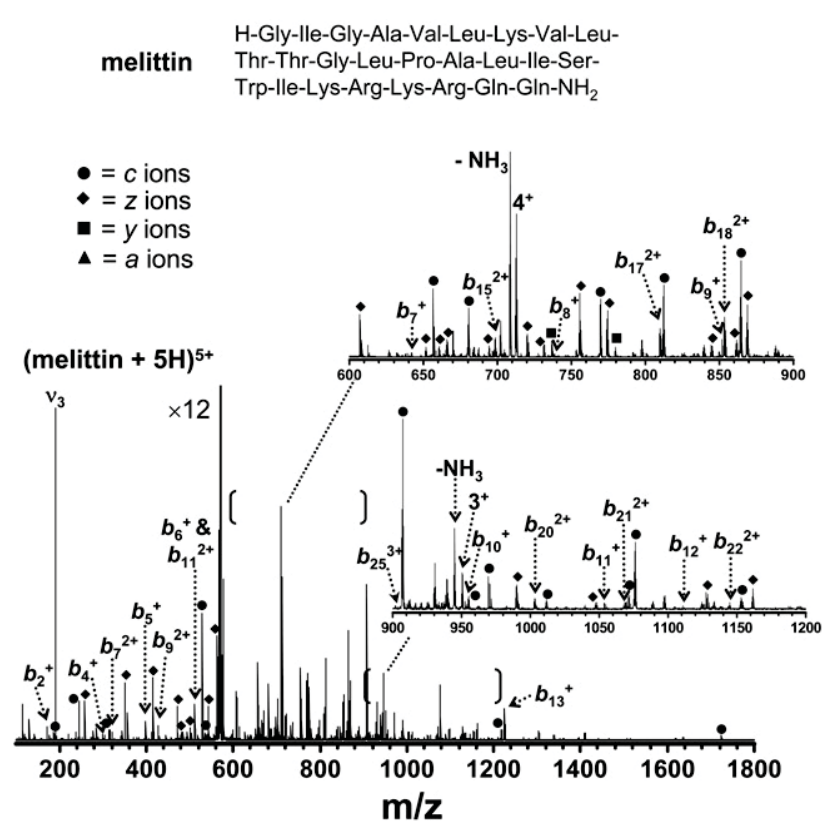

Figure 5. ECD spectrum from $[\text { melittin }+5 \mathrm{H}]^{5+}(100 \mathrm{~ms},-1 \mathrm{~V}$ bias voltage, 16 and 32 scans for $4+$ and $5+$, respectively). $\nu_{3}=$ third harmonic. 
be located at $\mathrm{K}^{7}$ while being solvated to $A V^{4-5}$. The fourth protonation site in quadruply charged melittin is likely to be the N-terminus. Thus, quadruply charged melittin is likely to have all four protons located at basic sites. However, for quintuply charged melittin, the chance is higher that one proton is located at a backbone amide nitrogen, which could account for the presence of many $b$-type ions in ECD of (melittin $+5 \mathrm{H})^{5+}$.

\section{Possible Mechanisms for the Formation of b-Type Ions}

Several mechanisms have been proposed for the formation of $b$-type ions in ECD $[8,11,14,16]$. In one of the proposed mechanisms, $b$-type ions are formed following $\mathrm{NH}_{3}$-loss from charge-reduced precursor ions. In our present work, such $\mathrm{NH}_{3}$ loss was found to be insignificant for MF, CCK and TrpSP (Figure 1a and Figure 2a and c) and nonexistent for Pep1 (Figure 2b). In addition, this reaction was observed for both VP and OT (Figure 4) but only the latter peptide yielded abundant $b$-type ions in ECD. These observations indicate that $b$-type ions are unlikely to be produced from $\mathrm{NH}_{3}$-loss precursors. The presence of $b$-type ions may also be associated with loss of $\mathrm{NH}_{3}$ from the corresponding c-type ions. However, Cooper has shown, based on double resonance experiments, that such a mechanism is unlikely [11] and she proposed an alternative mechanism in which electron capture by $[\mathrm{M}+$ $n \mathrm{H}]^{n+}$ precursor ions is followed by hydrogen atom loss, resulting in vibrationally excited even-electron [M $+(n-1) \mathrm{H}]^{(n-1)+{ }^{*}}$ ions that can dissociate via a mobile proton pathway, similar to CAD [11]. This mechanism is consistent with the data presented here. For example, odd-electron charge-reduced precursor ions were not observed in the ECD product ion spectra of MF (Figure 1a), CCK, Pep1, and TrpSP (Figure 2), that all contain abundant $b$-type ions. By contrast, both odd- and evenelectron charge-reduced precursor ions were seen for NMB and NMK (Figure $1 b$ and $c$ ) both of which yielded predominantly conventional ECD product ions. However, deviations from this behavior were found for VP (Figure 4a) and for LHRH (see Supplementary Figure). Here, even-electron charge-reduced precursor ions were almost exclusively observed although the ECD product ion spectra of these two peptides are characterized by abundant $c$ - and $z$-type ions.

An alternative explanation for the formation of $b$ type ions in ECD of nitrogen-protonated peptides has been proposed by Uggerud and coworkers [8]. These authors found from computational studies that electron capture by nitrogen-protonated $\mathrm{CH}_{3} \mathrm{CONH}_{2} \mathrm{CH}_{3}{ }^{+}$and subsequent dissociation of $\mathrm{CH}_{3} \mathrm{CONH}_{2} \mathrm{CH}_{3}{ }^{+}$should produce $b$ and $y$ rather than $c$ and $z$-type fragments. As discussed above, there is a high probability that the peptides exhibiting abundant $b$-type ions in their ECD spectra are protonated at an amide nitrogen. Thus, it seems plausible that the presence of abundant $b$-type ions in ECD for these peptides is due to initial charge neutralization taking place at amide nitrogens. One caveat with this hypothesis is that Uggerud et al. saw formation of $\mathrm{CH}_{3} \mathrm{CO}^{\bullet}$ and $\mathrm{NH}_{2} \mathrm{CH}_{3}$ from dissociation of $\mathrm{CH}_{3} \mathrm{CONH}_{2} \mathrm{CH}_{3}{ }^{\circ}$. For peptides, the former product would correspond to a radical $b$-type ion with a mass 1 $\mathrm{Da}$ (corresponding to one hydrogen) higher than that of an even-electron $b$ ion formed from vibrational excitation. However, all $b$ ions reported previously by others $[11,14,16]$ and here by us are even-electron species although, as stated above and shown in Figure 3, b-type ion formation in ECD and CAD is different. One possible mechanism for the formation of even-electron $b$ ions from radical $b$-type ions would be the existence of a long-lived $b^{\bullet} / y$ ion complex in which hydrogen migration may occur before separation of $b / y^{\bullet}$ type ions, similar to that proposed for $c / z$-type ions $[9,18$, 21]. If so, one would expect to see radical $y$-type ions in ECD spectra, which is rarely the case (one of two $y$-type ions from ECD of quintuply charged melittin has an isotopic distribution indicating a mixture of $y$ and $y^{\circ}$ ions in which $y^{\bullet}$ contains one additional hydrogen). On the other hand, in comparison to $b$-type ions, $y$-type ions are scarce in the ECD spectra presented here, indicating that radical $y$-type ions may rapidly undergo secondary fragmentation. It should be noted that evenelectron $y$-type ions have been proposed to form along with complementary radical $a$-type ions and CO [28]. Thus, even-electron $y^{\prime}$-type ions that are observed in most spectra may originate from a different process. We speculate that low-abundance $b$-type ions observed in ECD of peptides containing BAARs (e.g., LHRH and VP) may also originate from the co-existence of lowabundance nitrogen-protonated conformers.

\section{Conclusion}

The formation of $b$-type ions in ECD of peptides with few or no BAARs has been investigated in detail. It was found that the numbers and positions of BAARs as well as the charge state of peptides all affect the yield of $b$-type ions. In particular, abundant $b$-type ions were found for peptides without BAARs. A possible mechanism for the formation of $b$-type ions involves vibrationally excited even-electron charge-reduced precursor ions formed from hydrogen atom loss [11]. However, it is more likely that the presence of $b$-type ions for the peptides studied in this work is due to the existence of nitrogen-protonated conformers, which are present at a higher proportion for peptides with low basicity than for peptides with high basicity. We speculate that $b$-type ions sometimes found in ECD of peptides with high basicity may also be produced from low abundance nitrogen-protonated conformers.

\section{Acknowledgments}

This work was supported by the Searle Scholars Program, a Dow Corning Assistant Professorship, and the University of Michigan. 


\section{References}

1. Zubarev, R. A.; Kelleher, N. L.; McLafferty, F. W. Electron Capture Dissociation of Multiply Charged Protein Cations. A Nonergodic Process. J. Am. Chem. Soc. 1998, 120, 3265-3266.

2. Cooper, H. J.; Håkansson, K.; Marshall, A. G. The Role of Electron Capture Dissociation in Biomolecular Analysis. Mass Spectrom. Rev. 2005, 24, 201-222.

3. Zubarev, R. A. Reactions of Polypeptide Ions with Electrons in the Gas Phase. Mass Spectrom. Rev. 2003, 22, 57-77.

4. Håkansson, K.; Hudgins, R. R.; Marshall, A. G.; O'Hair, R. A. J. Electron Capture Dissociation and Infrared Multiphoton Dissociation of Oligodeoxynucleotide Dications. J. Am. Soc. Mass Spectrom. 2003, 14, $23-41$.

5. Adamson, J. T.; Håkansson, K. Electron Capture Dissociation of Oligosaccharides Ionized with Alkali, Alkaline Earth, and Transition Metals. Anal. Chem. 2007, 79, 2901-2910.

6. Budnik, B. A.; Haselmann, K. F.; Elkin, Y. N.; Gorbach, V. I.; Zubarev, R. A. Applications of Electron-Ion Dissociation Reactions for Analysis of Polycationic Chitooligosaccharides in Fourier Transform Mass Spectrometry. Anal. Chem. 2003, 75, 5994-6001.

7. Liu, H. C.; Lee, J. Y.; Sherman, D. H.; Håkansson, K. Collision Activated Dissociation, Infrared Multiphoton Dissociation, and Electron Capture Dissociation of the Bacillus anthracis Siderophore Petrobactin and Its Metal Ion Complexes. J. Am. Soc. Mass Spectrom. 2007, 18, 842-849.

8. Bakken, V.; Helgaker, T.; Uggerud, E. Models of Fragmentations Induced by Electron Attachment to Protonated Peptides. Eur. J. Mass Spectrom. 2004, 10, 625-638.

9. Belyayev, M. A.; Cournoyer, J. J.; Lin, C.; O'Connor, P. B. The Effect of Radical Trap Moieties on Electron Capture Dissociation Spectra of Substance P. J. Am. Soc. Mass Spectrom. 2006, 17, 1428-1436.

10. Breuker, K.; Oh, H. B.; Lin, C.; Carpenter, B. K.; McLafferty, F. W. Nonergodic and Conformational Control of the Electron Capture Dissociation of Protein Cations. Proc. Natl. Acad. Sci. U.S.A. 2004, 101, 14011-14016.

11. Cooper, H. J. Investigation of the Presence of b-Ions in Electron Capture Dissociation Mass Spectra. J. Am. Soc. Mass Spectrom. 2005, 16, 1932 1940.

12. Fung, Y. M. E.; Chan, T. W. D. Experimental and Theoretical Investigations of the Loss of Amino Acid Side Chains in Electron Capture Dissociation of Model Peptides. J. Am. Soc. Mass Spectrom. 2005, 16, 1523-1535

13. Fung, Y. M. E. Liu, H. Chan, T. W. D. Electron Capture Dissociation of Peptides Metalated with Alkaline-Earth Metal Ions. J. Am. Soc. Mass Spectrom. 2006, 17, 757-771.

14. Haselmann, K. F.; Schmidt, M. Do b-Ions occur from Vibrational Excitation Upon H-Desorption in Electron Capture Dissociation? Rapid Commun. Mass Spectrom. 2007, 21, 1003-1008.

15. Kleinnijenhuis, A. J.; Heck, A. J. R.; Duursma, M. C.; Heeren, R. M. A. Does Double Electron Capture Lead to the Formation of Biradicals? An ECD-SORI-CID Study on Lacticin $481 \mathrm{~J}$. Am. Soc. Mass Spectrom. 2005, $16,1595-1601$

16. Lee, S.; Chung, G.; I., K.; Oh, H. B. Electron Capture Dissociation Mass Spectrometry of Peptide Cations Containing a Lysine Homologue: A Mobile Proton Model for Explaining the Observation of b-Type Product Ions. Rapid Commun. Mass Spectrom. 2006, 20, 3167-3175.

17. Leymarie, N.; Costello, C. E.; O'Connor, P. B. Electron Capture Dissociation Initiates a Free Radical Reaction Cascade. J. Am. Chem. Soc. 2003, $125,8949-8958$

18. Lin, C.; O'Connor, P. B.; Cournoyer, J. J. Use of a Double Resonance Electron Capture Dissociation Experiment to Probe Fragment Intermediate Lifetimes J. Am. Soc. Mass Spectrom. 2006, 17, 1605-1615.

19. Liu, H.; Håkansson, K. Divalent Metal-Peptide Interactions Probed by Electron Capture Dissociation of Trications. J. Am. Soc. Mass Spectrom. 2006, 17, 1731-1741.

20. Mihalca, R.; Kleinnijenhuis, A. J.; McDonnell, L. A.; Heck, A. J. R.; Heeren, R. M. A. Electron Capture Dissociation at Low Temperatures Reveals Selective Dissociations. J. Am. Soc. Mass Spectrom. 2004, 15, 1869-1873.

21. O'Connor, P. B.; Lin, C.; Cournoyer, J. J.; Pittman, J. L.; Belyayev, M.; Budnik, B. A. Long-Lived Electron Capture Dissociation Product Ions Experience Radical Migration via Hydrogen Abstraction. I. Am. Soc. Mass Spectrom. 2006, 17, 576-585.

22. Patriksson, A.; Adams, C.; Kjeldsen, F.; Raber. Prediction of N-C $\mathrm{C}_{c}$ Bond Cleavage Frequencies in Electron Capture Dissociation of Trp-Cage Dications by Force-field Molecular Dynamics Simulations. Int. J. Mass Spectrom. 2006, 248, 124-135.
23. Sobczyk, M.; Anusiewicz, W.; Berdys-Kochanska, J.; Sawicka, A.; Skurski, P.; Simons, J. Coulomb-Assisted Dissociative Electron Attachment: Application to a Model Peptide. J. Phys. Chem. A 2005, 109, 250-258.

24. Syrstad, E. A.; Turecek, F. Toward a General Mechanism of Electron Capture Dissociation. J. Am. Soc. Mass Spectrom. 2005, 16, 208-224.

25. Tsybin, Y. O.; Haselmann, K. F.; Emmett, M. R.; Hendrickson, C. L. Marshall, A. G. Charge Location Directs Electron Capture Dissociation of Peptide Cations. J. Am. Soc. Mass Spectrom. 2006, 17, 1704-1711.

26. Turecek, F. N-C $\mathrm{C}_{\alpha}$ Bond Dissociation Energies and Kinetics in Amide and Peptide Radicals. Is the Dissociation a Nonergodic Process? J. Am. Chem. Soc. 2003, 125, 5954-5963.

27. Zubarev, R. A.; Haselmann, K. F.; Budnik, B.; Kjeldsen, F.; Jensen, F.Towards an Understanding of the Mechanism of Electron Capture Dissociation: A Historical Perspective and Modern Ideas. Eur. Mass Spectrom. 2002, 8, 337-349.

28. Zubarev, R. A.; Kruger, N. A.; Fridriksson, E. K.; Lewis, M. A.; Horn, D. M.; Carpenter, B. K.; McLafferty, F. W. Electron Capture Dissociation of Gaseous Multiply-charged Proteins is Favored at Disulfide Bonds and Other Sites of High Hydrogen Atom Affinity. J. Am. Chem. Soc. 1999, 121, 2857-2862.

29. Chen, X. H.; Turecek, F. The Arginine Anomaly: Arginine Radicals are Poor Hydrogen Atom Donors in Electron Transfer Induced Dissociations. J. Am. Chem. Soc. 2006, 128, 12520-12530.

30. Chakraborty, T.; Holm, A. I. S.; Hvelplund, P.; Nielsen, S. B.; Poully J. C.; Worm, E. S.; Williams, E. R. On the Survival of Peptide Cations after Electron Capture: Role of Internal Hydrogen Bonding and Microsolvation J. Am. Soc. Mass Spectrom. 2006, 17, 1675-1680.

31. Iavarone, A. T.; Paech, K.; Williams, E. R. Effects of Charge State and Cationizing Agent on the Electron Capture Dissociation of a Peptide. Anal. Chem. 2004, 76, 2231-2238.

32. Leib, R. D.; Donald, W. A.; Bush, M. F.; O'Brien, J. T.; Williams, E. R Internal Energy Deposition in Electron Capture Dissociation Measured using Hydrated Divalent Metal Ions as Nanocalorimeters. J. Am. Chem. Soc. 2007, 129, 4894-4895.

33. Robinson, E. W.; Leib, R. D.; Williams, E. R. The Role of Conformation on Electron Capture Dissociation of Ubiquitin. J. Am. Soc. Mass Spectrom. 2006, 17, 1469-1479.

34. Jones, J. W.; Sasaki, T.; Goodlett, D. R.; Turecek, F. Electron Capture in Spin-trap Capped Peptides. An Experimental Example of Ergodic Dissociation in Peptide Cation-Radicals. J. Am. Soc. Mass Spectrom. 2007, 18, 432-444.

35. Savitski, M. M.; Kjeldsen, F. Nielsen, M. L. Zubarev, R. A. Hydrogen Rearrangement to and from Radical z Fragments in Electron Capture Dissociation of Peptides. J. Am. Soc. Mass Spectrom. 2007, 18, 113-120.

36. Yang, J.; Mo, J.; Adamson, J. T.; Håkansson, K. Characterization of Oligodeoxynucleotides by Electron Detachment Dissociation Fourier Transform Ion Cyclotron Resonance Mass Spectrometry. Anal. Chem. 2005, 77, 1876-1882.

37. de Koning, L. J.; Nibbering, N. M. M.; van Orden, S. L.; Laukien, F. H. Mass Selection of Ions in a Fourier Transform Ion Cyclotron Resonance Trap using Correlated Harmonic Excitation Fields (CHEF). Int. J. Mass Spectrom. 1997, 165, 209-219.

38. Caravatti, P.; Allemann, M. The Infinity Cell-a New Trapped Ion Cell with Radiofrequency Covered Trapping Electrodes for Fourier Transform Ion Cyclotron Resonance Mass Spectrometry. Org. Mass Spectrom. 1991, 26, 514-518.

39. Tsybin, Y. O.; Witt, M.; Baykut, G.; Kjeldsen, F. Håkansson, P. Combined Infrared Multiphoton Dissociation and Electron Capture Dissociation with a Hollow Electron Beam in Fourier Transform Ion Cyclotron Resonance Mass Spectrometry. Rapid Commun. Mass Spectrom. 2003, 17, 1759-1768.

40. Senko, M. W.; Canterbury, I. D.; Guan, S.; Marshall, A. G. A HighPerformance Modular Data System for FT-ICR Mass Spectrometry. Rapid Commun. Mass Spectrom. 1996, 10, 1839-1844.

41. Ledford, E. B.; Jr.; Rempel, D. L.; Gross, M. L. Space Charge Effects in Fourier Transform Mass Spectrometry Mass Calibration. Anal. Chem. 1984, 56, 2744-2748.

42. Paizs, B.; Suhai, S. Fragmentation Pathways of Protonated Peptides. Mass Spectrom. Rev. 2005, 24, 508-548.

43. Kjeldsen, F.; Savitski, M. M.; Adams, C. M.; Zubarev, R. A. Determination of the Location of Positive Charges in Gas-Phase Polypeptide Polycations by Tandem Mass Spectrometry. Int. J. Mass Spectrom. 2006, $252,204-212$

44. Kweon, H. K.; Håkansson, K. Site-Specific Amide Hydrogen Exchange in Melittin Probed by Electron Capture Dissociation Fourier Transform Ion Cyclotron Resonance Mass Spectrometry. Analyst 2006, 131, 275280. 